\title{
A SEQUENTIAL DETECTION METHOD FOR LATE AUDITORY EVOKED POTENTIALS
}

\author{
U. Hoppe, U. Eysholdt \\ Abteilung für Phoniatrie und Pädaudiologie \\ Universitäts-HNO-Klinik \\ Friedrich-Alexander Universität \\ Erlangen, Germany
}

\begin{abstract}
This work presents a novel mechanism for detection of late auditory evoked potentials (AEP). AEPs, which are an important diagnostic tool to detect hearing deficiencies, are contained within the electroencephalogram (EEG) at a very low SNR. Our proposed automatic detection of AEPs is based on a Wavelet-Transform of EEG data for feature extraction. Several transform coefficients are then used for a classification by a neural network; its decisions on successive EEG segments are judged by a sequential statistical test. This test stops when a certain confidence is reached to either accept or reject an $A E P$. Besides providing an objective recognition of $A E P$, this procedure can considerably reduce measurement time.
\end{abstract}

\section{INTRODUCTION}

Late auditory evoked potentials (AEP) are part of the electroencephalogram (EEG) in reaction to the cortical processing of perceived acoustic stimuli. Therefore, in audiometry late AEPs are widely used to obtain objective frequency specific hearing thresholds, with objectivity in a sense that the active cooperation of the experimentee is replaced by the interpretation of the EEG by an operator. As due to background EEG the AEP often has an SNR below $-10 \mathrm{~dB}$, this interpretation is very difficult and to date had to be based on the support by techniques like eg. averaging of sweeps (EEG segments synchronously recorded to the stimuli), cross-correlation, or Fourier coefficients [1].

In the following, we introduce a new method to sequentially assess successive sweeps in three stages as it can be seen in Fig. 1, comprising of a feature extraction by a discrete wavelet transformation (DWT) of the sweeps, which yields a good parameterisation of the AEP pattern by a set of few transform coefficients $[2,3]$, followed by neural network (NN) classification of these coefficients for successive sweeps. However, as the NN possesses a high
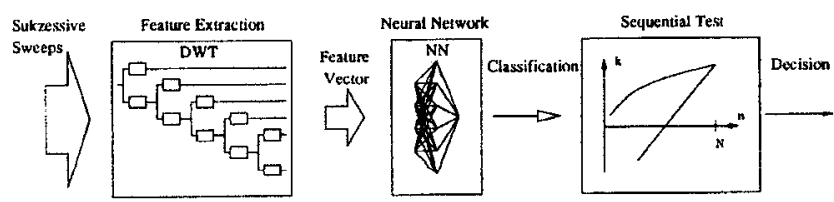

Figure 1: Block diagram of AEP detection scheme

\author{
S. Weiß \\ Signal Processing Division \\ Dept. of Electronic and Electrical Eng. \\ University of Strathclyde \\ Glasgow, Scotland
}

probability for false positives (type-II-error), a sequential statistical test judging the number of positive or negative classifications produced by the NN forms the last stage of the detection procedure. This sequential test allows a decision based on a predefined confidence, and stops the measurement when this confidence is reached.

\section{METHODS}

\subsection{Feature Extraction Procedure}

A typical late AEP of a person with normal hearing ability is shown in Fig. 2(a), with a low frequent response in the range of approx. $3-5 \mathrm{~Hz}$ and a duration of up to $400 \mathrm{~ms}$ after the stimulus [1]. Variations from this shape must be expected for children and hearing impaired. Generally, a lower level of the acoustic stimulus yields a less significant amplitude and larger latency of the main peak, causing the AEP to be much harder to detect.

To parameterise the basic features in the recorded EEG segments (sweeps), a discrete Wavelet transform (DWT) has been successfully employed [3], based on the general use of the DWT for evoked potentials [2]. Using Mallat's Algorithm [4], every sweep is decomposed by an octave filter bank, where the subbands yield the wavelet transform coefficients. For our measurements, the expected time-frequency range of the AEP is represented by 7 DWT coefficients in total, forming the feature vector $(\mathrm{FV})$. An example of the information contained within the FV is given in Fig. 2(b), showing the inverse DWT of the feature vector corresponding to the curve in Fig. 2(a). The advantages of applying a DWT are the noise reduction by cutting off irrelevant frequencies, the immense data reduction, and the low variance between individual feature vectors as each coefficient represents a time interval of more than $100 \mathrm{~ms}$.
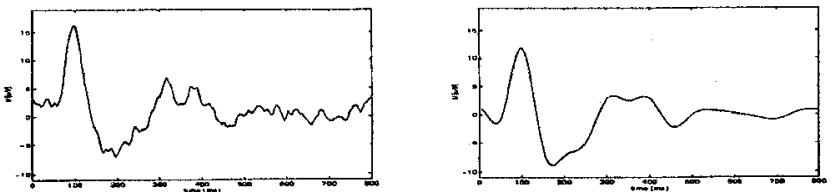

Figure 2: (a) typical late AEP after averaging of 50 single sweeps, (b) reconstruction of DWT coefficients in the feature vector. 


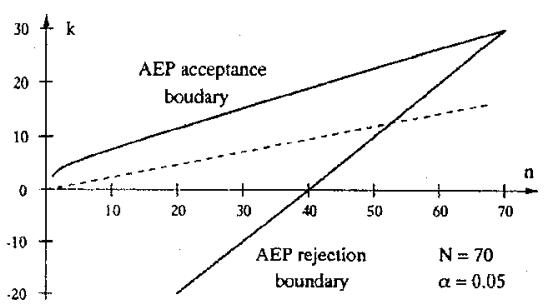

Figure 3: Decision based on the acceptance and rejection boundaries of the sequential test of NN classification

\subsection{Neural Network Classification of Single Fea- ture Vectors}

An NN is used to binary classify the previously calculated FV of a single sweep to identify a potential AEP. The FV is fed into a feed-forward NN with one hidden layer, after removing the mean and a normalisation of the FV's $L_{\infty}$ " norm to one. Simulations suggest an optimum number of 8 neurons in the hidden layer. With fewer neurons, the classification results decrease, while an increase in hidden neurons only slightly improves the classification at the expense of a reduced generalisation of the NN. Considering the potential variations of the AEP, generalisation is an important characteristic of the NN.

Training of the NN was performed with 200 feature vectors which have been calculated from both averaged measurements of 15 experimentees and synthetic signals produced from expert knowledge.

Due to normalisation, all input FV lie on the surface of a 7-dimensional hypercube. Using Monte-Carlo integration with $10^{4}$ random input vectors, the surface area for positive classification was determined to be $24 \%$. As this false positive rate is too large, the decision cannot be based upon the classification of a single sweep's FV.

\subsection{Sequential Statistical Analysis}

To improve the decision, the classification of single sweeps produced by the $\mathrm{NN}$ is sequentially tested using a statistical method described in [5]. This test checks if the number of correctly classified sweeps is outside the expected statistical distribution for random values. The significance $\alpha$ of this test, which is identical to the typeII-error, and the maximum number of measurements $N$ have to be specified in advance.

For practical reasons, we have chosen $N=70$ and $\alpha=0.05$. The results of the single sweep classification can be regarded as a binomial distribution $B_{n p}(k)$ where $B$ denotes the probability to get $k$ positive classifications out of a total number of $n$, and $p$ is the probability for a positive classification. For $n>10, B_{n p}(k)$ approximates a Gaussian distribution with mean $\bar{a}=n p$ and variance $\sigma^{2}=n p(1-p)$, such that $5 \%$-percentiles of the distribution can be calculated in dependency of $n$. Upon these, boundaries for acceptance or rejection of an AEP can be set, as shown in Fig. 3. There, the abscissa represents the number of measurements, $n$, the ordinate the total num-

\begin{tabular}{|l||c|c|c|c|c|}
\hline stimulus level [dB] & 20 & 40 & 60 & 80 & $-\infty^{*}$ \\
\hline rate of positives & 0.80 & 0.96 & 0.94 & 0.98 & 0.06 \\
\hline average no. sweeps & 34 & 27 & 25 & 22 & 49 \\
\hline
\end{tabular}

Table 1: Detection rates and average number of sweeps need for a decision for various stimulus levels (" no stimulus given).

ber of positives, $k$. If after $n$ sweeps $k$ exceeds the upper boundary, an AEP is detected and the measurement can be stopped. When the lower boundary is reached after $n$ measurements it is impossible that the upper boundary is met in the remaining $N-n$ sweeps, an AEP is rejected and the measurement can also be stopped. The dashed line shows the mean value ( $n p$ with $p=0.24$ ) for positive NN-classifications in the absence of an AEP.

\section{R.ESULTS}

The proposed method has been applied to 232 sets of EEG measurements of eight normal hearing adults, which have been acoustically stimulated by $1000 \mathrm{~Hz}$ short sinebursts of various sound pressure levels. The results are listed in Tab. 1, together with the average number of sweeps needed for a decision. Results for 48 measurement sets without any given stimulus show a very low false decision rate for an incorrectly assumed AEP.

\section{CONCLUSION}

This work has introduced an automatic detection method for late AEPs, based on a DWT for low noise feature extraction, a feature classification using a neural network, and a decision mechanism based on a sequential statistical assessment of the NN's output. Practical results underline the suitability of this method. Additionally it was shown that substantial reduction of measurement time can be achieved when sequential testing is applied. Thus, an audiometric investigation via AEP becomes more accurate and faster.

\section{REFERENCES}

[1] S. Hoth and C. Weber. "Kritische Wertung der Hörschwellenbestimmung mittels der Hirnrindenpotentiale, Teil 1". Audiol. Akust.,190-200, May 1990.

[2] E. Bartnik, K. Blinowska, and P. Durka. "Single Evoked Potential Reconstruction by Means of Wavelet Transform". Biol. Cyb.,67:175-181,1992.

[3] S. WeiB and U. Hoppe. "Recognition and Reconstruction of Late Auditory Evoked Potentials Using Wavelet Analysis". To be pres. at 3rd Symp. TimeFreq. Time-Scale Anal., Paris, June 1996.

[4] S. G. Mallat. "A Theory for Multiresolution Signal Decomposition: The Wavelet Representation". IEEE Trans PAMI, 11(7):674-692, July 1989.

[5] R. G. Miller. "Sequential Signed-Rank Test". J. Am. Stat. Ass., 65(332):1554-1561, Dec. 1970. 